\title{
Bi-allelic variants in MTMR5/SBF1 cause Charcot-Marie-Tooth type 4B3 featuring mitochondrial dysfunction
}

\author{
Beatrice Berti ${ }^{5 \dagger}$, Giovanna Longo ${ }^{2 \dagger}$, Francesco Mari ${ }^{1}$, Stefano Doccini ${ }^{3}$, Ilaria Piccolo ${ }^{2}$, Maria Alice Donati ${ }^{4}$, \\ Francesca Moro ${ }^{3}$, Renzo Guerrini ${ }^{1}$, Filippo M. Santorelli $3^{*}$ (D) and Vittoria Petruzzella ${ }^{2^{*}}$ (])
}

\begin{abstract}
Background: Charcot-Marie-Tooth disease (CMT) type 4B3 (CMT4B3) is a rare form of genetic neuropathy associated with variants in the MTMR5/SBF1 gene. MTMR5/SBF1 is a pseudophosphatase predicted to regulate endo-lysosomal trafficking in tandem with other MTMRs. Although almost ubiquitously expressed, pathogenic variants primarily impact on the peripheral nervous system, corroborating the involvement of MTMR5/SBF1 and its molecular partners in Schwann cells-mediated myelinization.
\end{abstract}

Case presentation: We report a case of severe CMT4B3 characterized by early-onset motor and axonal polyneuropathy in an Italian child in absence of any evidence of brain and spine MRI abnormalities or intellectual disability and with a biochemical profile suggestive of mitochondrial disease. Using an integrated approach combining both NGS gene panels and WES analysis, we identified two novel compound heterozygous missense variants in MTMR5/SBF1 gene, p.R763H (c.2291G > A) and p.G1064E (c.3194G > A). Studies in muscle identified partial defects of oxidative metabolism.

Conclusion: We describe the first case of an early onset severe polyneuropathy with motor and axonal involvement, due to recessive variants in the MTMR5/SBF1 gene, with no evidence of brain and spine MRI abnormalities, intellectual disability, no clinical and neurophysiological evidences of distal sensory impairment, and rapid neuromuscular deterioration. This report suggests that MTMR5/SBF1 should be considered in cases of infantile-onset CMT with secondary mitochondrial dysfunction.

Keywords: MTMR5/SBF1, Charcot-Marie-Tooth 4B3, Mitochondrial diseases, Next-generation sequencing, Case report

\section{Background}

Charcot-Marie-Tooth disease (CMT) is one of the most common inherited neurological disorders [1]. It is characterized by chronic motor and/or sensory polyneuropathy

*Correspondence: filippo3364@gmail.com; vittoria.petruzzella@uniba.it †Beatrice Berti and Giovanna Longo contribute equally to the work

${ }^{2}$ Department of Medical Basic Sciences, Neurosciences and Sense Organs, University of Bari Aldo Moro, Piazza G. Cesare, 11, 70124 Bari, Italy

${ }^{3}$ IRCCS Fondazione Stella Maris, via dei Giacinti 2, Calambrone,

56128 Pisa, Italy

Full list of author information is available at the end of the article with progressive degeneration of muscles in the extremities and by impaired motor and sensory function [2]. Classification has been based on the pattern of inheritance together with the findings of nerve conduction velocity (NCV) studies and nerve biopsy analysis [3], and this has led to the definition of two major subgroups: axonal and demyelinating forms [4].

CMT4B is among the rarest autosomal recessive demyelinating forms of the disease with fewer than a hundred CMT4B cases reported, mostly coming from countries with high rates of consanguineous marriage [5]. It is 
subdivided into the three clinically and genetically distinct subtypes CMT4B1, CMT4B2 and CMT4B3, caused by variants in myotubularin-related proteins, namely MTMR2, MTMR13/SBF2 and MTMR5/SBF1, respectively, mainly involved in regulating endolysosomal trafficking [6]. The almost exclusive peripheral nervous system (PNS) involvement seen in CMT4B suggests that myelinating Schwann cells are extremely sensitive to disruption of endolysosomal trafficking $[7,8]$. However, it is well established that mutations of myelin components can impact on their axonal counterpart due to rearrangements of protein in the axonal membrane, axonal atrophy and even axonal loss [9].

Mitochondrial diseases (MDs) are heterogeneous conditions resulting from a primary dysfunction of the oxidative phosphorylation (OxPhos) system caused by alterations in either nuclear (nDNA) or mitochondrial (mtDNA) genomes. Peripheral neuropathy is often among the large array of features characterizing MDs associated with defects in genes related to mtDNA maintenance/replication or to ATP synthesis, and in several nDNA-encoded proteins. In most cases, peripheral neuropathy occurs in the setting of multisystemic neurological manifestations, and more rarely as the manifestation at onset [10]. Chronic sensorimotor axonal polyneuropathy is the predominant pattern, whereas demyelinating patterns are less common [11-13]. The fact that MD-like biochemical features are frequent both in non-canonical forms of peripheral neuropathy and in other neuromuscular disorders highlights the critical role played by oxidative metabolism in muscle and nerve function $[10,14]$.

To date, only five families harboring variants in MTMR5/SBF1 have been described [15-19] with age at onset of symptoms ranging between a few months and a few years. Clinical and imaging phenotypes are polymorphic: cases may or may not show developmental delay, facial dysmorphisms, and abnormal brain and spinal MRI. Similarly, electrophysiological studies are heterogeneous, revealing mainly demyelinating forms of neuropathy.

We report the case of an Italian girl with progressive and severe infantile-onset axonal motor neuropathy and biochemical features suggestive of mitochondrial disease in whom next-generation sequencing studies allowed us to identify biallelic variants in MTMR5/SBF1.

\section{Case presentation}

This 12-year-old Italian girl with an unremarkable family and prenatal history presented bilateral congenital talonvalgus-pronated clubfoot at birth and slight neuromotor developmental delay from the age of 18 months. Her first neurological evaluation at the age of 4 years showed global hypotonia, marked hyperlordosis, anserine gait,
Gowers' sign, easy fatigability, knee valgus and absence of osteotendinous reflexes. Electroneurography disclosed severe motor polyneuropathy with predominant axonal features, diffuse reduction of compound action muscular potentials (CMAP, values under $1.5 \mathrm{mV}$ ) and only slight decrement of NCV (38-43 m/s). Sensory action potentials and brain and spinal cord MRI were normal. Gene testing for genomic rearrangements of $P M P 22$ and punctuate variants in MNF2 and GDAP1 were negative. Follow-up electrophysiological evaluations over time showed a slowly progressive reduction of motor action potential amplitude (values under $0.5 \mathrm{mV}$ ) with evidence of diffuse denervation activity on electromyography suggesting a neurogenic pattern. Several cognitive evaluations were performed, and all were normal. From the age of 6 years, the patient showed a progressive deterioration, with worsening of ambulation and of respiratory function, to the point of requiring non-invasive positive pressure ventilation, and the development of dysphagia, necessitating G-tube placement. Independent ambulation was lost at the age of 9 , when she began needing walking aids; she became wheelchair-dependent at the age of 11 . The patient showed no cognitive impairment during the clinical course, and at the latest follow up (age 11), brain and spinal MRI remained normal.

The progression of the neuromuscular involvement (both clinical and neurophysiological) in this child, in the absence of any metabolic abnormality in blood tests, prompted us to perform a muscle biopsy. This showed a normal histochemical pattern with wiped out areas on oxidative metabolism stains, while NADH

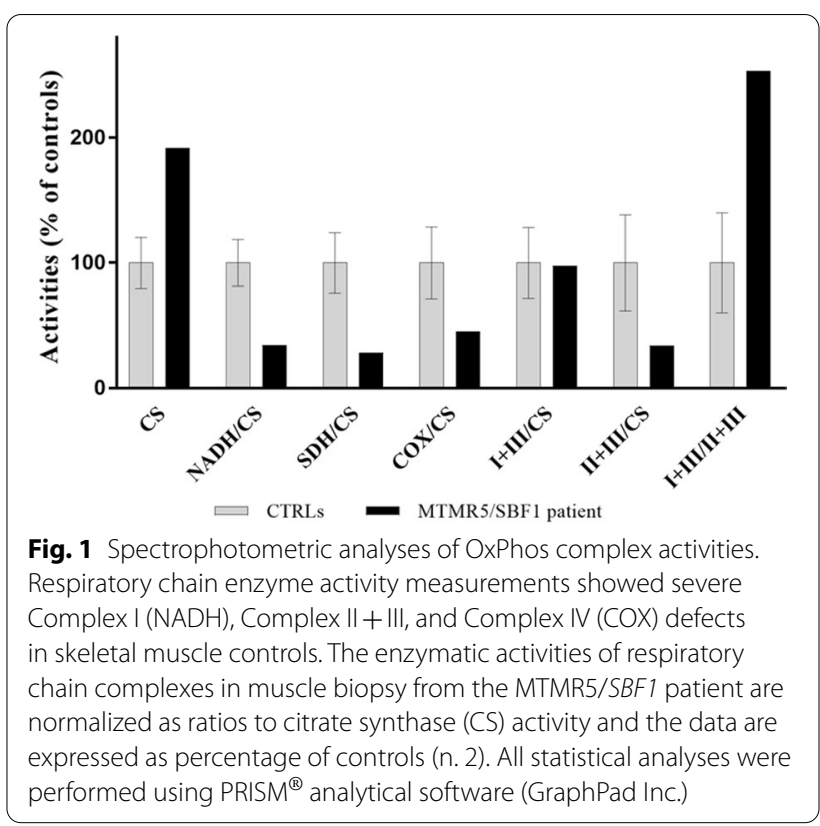


a

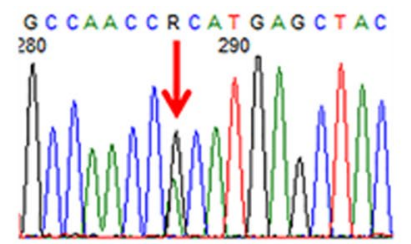

Ex 19 c.2291G>A (p.R763H)

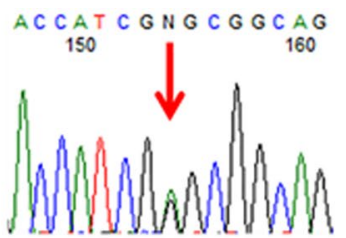

Ex 25 c.3194G >A (p.G1064E)

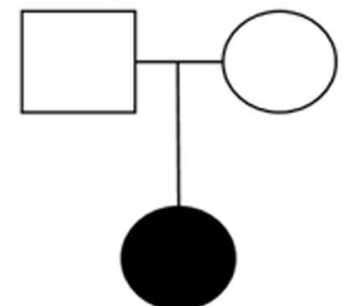

GC CAACCRCATGAGCTAC

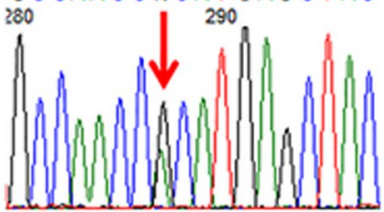

c. $2291 \mathrm{G}>\mathrm{A}(\mathrm{p} . \mathrm{R} 763 \mathrm{H})$

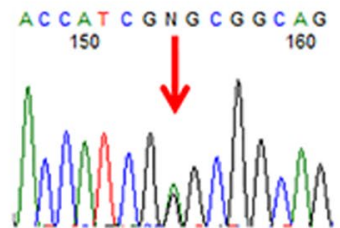

c.3194G $>$ A (p.G1064E)

b

\begin{tabular}{|cccccc|}
\hline Mutation & SIFT & PolyPhen-2 & dbSNPID & NHLBI ESP & ExAC Frequency \\
\hline p.R763H & Damaging & Probably Damaging & 200624784 & 0.01 & 0 \\
p.G1064E & Tolerated & Probably Damaging & 201200122 & 0.01 & 0.01 \\
\hline
\end{tabular}

Fig. 2 Novel compound heterozygous missense variants in the MTMR5/SBF1 patient. a Familial pedigree and Sanger sequencing data for the MTMR5/SBF1 patient and her parents demonstrating recessive inheritance of compound heterozygous c.2291G > A (p.R763H) and c.3194G > A (p.G1064E) mutations of MTMR5/SBF1 (the nomenclature of variant refers to NM_001365819.1). The arrows indicate the variant sites. $\mathbf{b}$ Predictions of the pathogenic effects of both variants as analyzed using the following prediction tools: SIFT [27]; PolyPhen-2 [28]; dbSNP [29]; NHLBI ESP [30]; GnomAD v3.1 at http://genome.ucsc.edu

staining showed the presence of predominant hypertrophic fibers, suggesting an alteration of the myofibrillar framework. Overall, the results pointed to significant neurogenic muscular atrophy (Additional file 1: Figure S1). Spectrophotometric determination of OxPhos activities in muscle homogenate showed multiple defects of the respiratory chain complexes with high levels of citrate synthetase, an index of mitochondrial proliferation. In particular, these studies [20] showed Complex I (NADH: ubiquinone oxidoreductase), Complexes II + III (succinate: cytochrome $c$ reductase) and Complex IV (cytochrome $c$ oxidase) activity to be 65, 55 and $66 \%$ of normal values, respectively (Fig. 1). Estimation by realtime PCR showed that total mtDNA content was also lower than normal values, as was the mtDNA level in muscle (about $30 \%$ of normal control values), whereas the mtDNA/nDNA ratio [21] in cultured skin fibroblasts was normal.

Having excluded pathogenic alterations in over 1000 mitochondrial genes by massive gene testing (MitoExome analysis as in [22]), we identified bi-allelic variants in MTMR5/SBF (NM_001365819.1) by whole-exome 


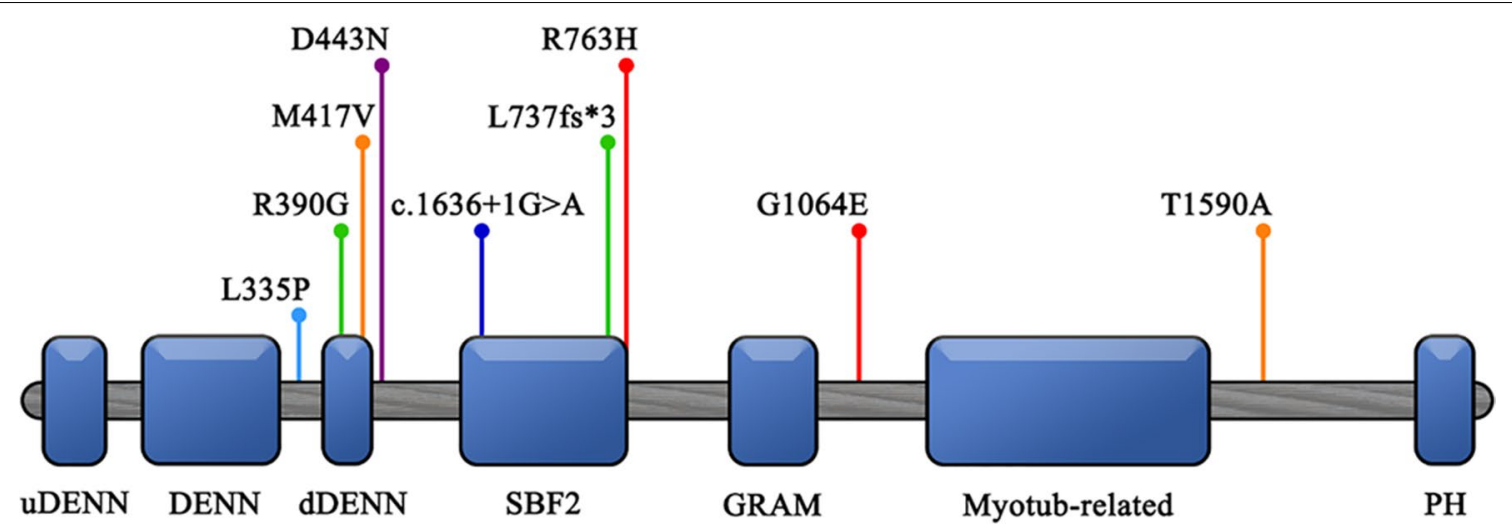

Fig. 3 Scheme of the SBF1 protein secondary structure. upstream DENN (UDENN), differentially expressed in normal and neoplastic cells (DENN) and downstream DENN (dDENN) domains, SBF2 domain, Glucosyltransferases, Rab-like GTPase activators and Myotubularins (GRAM) domain, Myotubularin-like phosphatase domain and PH (Pleckstrin Homology) domain. Pins highlight all reported variants: light blue—Romani et al. [19]; green—Manole et al. [17]; orange—Nakhro et al. [18]; purple—Alazami et al. [15]; blue—Flusser et al. [16]; red_our variants

sequencing (WES) of the family trio; this was done by precise filtering and prioritization of over 740 variants in more than 600 rare genes with a customized in-house bioinformatic pipeline using the criteria as reported in [23]. Sanger sequencing confirmed that the patient harbored the p.R763H (c.2291G > A) variant, inherited from her father, and the p.G1064E (c.3194G > A) variant, inherited from her mother (Fig. 2). According to the ACMG guidelines [24], these variants were classified as "uncertain" (evidence of pathogenicity: PM2, PP3) and "likely benign", respectively, with CADD scores of 29.3 (p.R763H) and 24.2 (p.G1064E). The variants were absent in 175 CMT patients collected in our lab as well as in inhouse WES data from 164 cases.

\section{Discussion and conclusions}

CMT4B3, ranging from pure demyelinating neuropathy with myelin outfolding to axonal forms complicated by multiple cranial involvement, intellectual disability, microcephaly and dysmorphic features, may show various clinical pictures, from mild to more severe. Herein, we further broaden the spectrum of CMT4B3 phenotypes by reporting a case of severe and progressive polyneuropathy with axonal motor involvement in a child without cognitive impairment and with normal MRI findings. We identified two variants in MTMR5/ SBF1 by massive sequencing. The p.R763H mapped on the edge of the SBF1/SBF2 domain of MTMR5 (Fig. 3), a 220-amino acid motif in the middle of both the SBF1 and SBF2 proteins [25]. Although two other variants have been reported in this specific motif [16, 17], its function remains to be ascertained. The second variant, p.G1064E, mapped within a linker region located between the GRAM and myotubularin-like phosphatase domains (Fig. 3).The p.G1064E variant is predicted in silico as tolerated and it might have a moderate impact on the structure/function of the protein. As in our case, less severe missense variants within the linker regions (Fig. 3) were described in three Korean sisters presenting progressive demyelinating sensorymotor neuropathy with myelin outfolding and slow disease progression without cognitive impairment [18]. Conversely, all the remaining CMT4B3 cases harbored pathogenic variants predicting a high functional impact on protein structure $[15,17,19,26]$.

It is interesting that the initial clinical and laboratory investigations in our child suggested a possible mitochondrial role with predominant PNS involvement, a non-canonical, yet at the same time not particularly rare, MD presentation [12]. It is possible that accumulation of toxic metabolites or secondary impairment of the OxPhos machinery and mitochondrial metabolism, or a combination of the two, might affect peripheral nerves and long axons leading to atypical mitochondrial presentations [10-13]. However, these issues require more precise investigations in peripheral nerves.

In conclusion, we identified two novel missense in MTMR5/SBF1. This finding underlines the importance of testing this gene in children with clinical evidence of PNS involvement, even if they present no associated neurodevelopmental features or skeletal abnormalities. Whilst our case report further corroborates the importance of WES studies as a first-tier diagnostic method in children with complex neurological phenotypes, it also highlights the presence of secondary features of MD, and thus the need for better studies on the involvement of disturbed oxidative metabolism in chronic inherited neuropathies. 


\begin{abstract}
Abbreviations
CMT: Charcot-Marie-Tooth disease; NCV: Nerve conduction velocity; PNS: Peripheral nervous system; MDs: Mitochondrial diseases; OxPhos: Oxidative phosphorylation; nDNA: Nuclear DNA; mtDNA: Mitochondrial DNA; ACMG: American College of Medical Genetics.
\end{abstract}

\section{Supplementary Information}

The online version contains supplementary material available at https://doi. org/10.1186/s12920-021-01001-1.

\section{Additional file 1: Figure S1. Staining in muscle biopsy from a} MTMR5/SBF1 patient. a Muscle-specific staining with hematoxylin and eosin (HE) to show the myofibril morphology; b Gomori trichrome staining showing the intermyofibrillar network; c ATPase stain at $\mathrm{pH} 4.3$ for type 1 myofibers; d NADH staining for respiratory complex I activity; predominant hypertrophic fibers can be seen in the intermyofibrillar network. We used a Zeiss AxioVision microscope and an AxioVision software for data collection. $\mathrm{Bar}=50 \mu$.

\section{Acknowledgements}

This paper is dedicated to the loving memory of AP who recently passed away. We thank the family for participating in this study, the patients'association MITOCON and UILDM (Unione Italiana Lotta alla Distrofia Muscolare) and CollaGe-associazione-Genitori-Manzoni-Poli-Molfetta. We are indebted to Dr. Catherine J. Wrenn for the expert editing of the manuscript and critical advice.

\section{Authors' contributions}

BB: clinical characterization; writing the manuscript. GL: genetic and molecular studies; Editing the manuscript. FMa: clinical characterization; writing the manuscript. SD: genetic and molecular studies. IP: genetic and molecular studies; editing the manuscript. MAD: clinical characterization; editing the manuscript. FMo: WES studies. RG: supervision of clinical aspects. FMS: obtaining funds; supervising the project. VP: genetic and molecular studies; initiated and supervised the project. All authors: data analysis, experimental design. All the authors gave final approval of the version to be published and agreement to be accountable for all aspects of the work in ensuring that questions related to the accuracy or integrity of any part of the work are appropriately investigated and resolved.

\section{Funding}

This study received partial financial support from the Italian Ministry of Health-Ricerca Corrente, MITO-NEXT, Mit-OMICS (to FMS) and 5X1000 (to FM), Fondazione Telethon (Grant numbers GUP09004). This work was supported by grants from Regione Puglia-Malattie Rare-Petruzzella Del. N.246 10.09.2019_ UPB-SMBNOS and by donations of Parents' Associations (to VP).

\section{Availability of data and materials}

The datasets generated and analyzed during the current study has been registered with the BioProject database with the ID PRJNA733011 (http://www. ncbi.nlm.nih.gov/bioproject/733011). Predictably or probably deleterious scores were determined using an in-silico pipeline employing SIFT (https:// sift.bii.a-star.edu.sg/), PolyPhen-2 (http://genetics.bwh.harvard.edu/pph2/); dbSNP (https://www.ncbi.nlm.nih.gov/snp/); NHLBI ESP (https://evs.gs.washi ngton.edu/EVS/); GnomAD v3.1 (http://genome.ucsc.edu). Details of the variants analyzed during the current study are deposited in ClinVar (https:// www.ncbi.nlm.nih.gov/clinvar/) under the accession codes: RCV001449576.1 (p.Arg763His) and RCV001449656.1 (p.Gly1064Glu). Sanger sequencing was run using the set of primers: Sbf1-19F 5'-TATGGGGATGTGCAGACTCA-3'/Sbf119R 5'-ACTCTCCGTGCAGACCTTGT-3'; Sbf1-25F 5'-TCCCTCAGGTATGGATCT GG-3'/Sbf1-25R 5'-CTCCCTGGCCAATGTCAG-3'

\section{Declarations}

\section{Ethics approval and consent to participate}

This study was approved by the Tuscany Regional Pediatric Ethics Committee. All the procedures complied with the Helsinki Declaration of 1975. Genetic studies were performed after parental written informed consent had been obtained.

\section{Consent to publish}

Written informed consent was received for publication of clinical data and brain imaging results from the patient's parents.

\section{Competing interests}

The authors declare that they have no competing interests.

\section{Author details}

${ }^{1}$ Child Neurology Unit, Meyer Children's Hospital, Florence, Italy. ${ }^{2}$ Department of Medical Basic Sciences, Neurosciences and Sense Organs, University of Bari Aldo Moro, Piazza G. Cesare, 11, 70124 Bari, Italy. ${ }^{3}$ IRCCS Fondazione Stella Maris, via dei Giacinti 2, Calambrone, 56128 Pisa, Italy. ${ }^{4}$ Metabolic Unit, Meyer Children's Hospital, Florence, Italy. ${ }^{5}$ Present Address: Pediatric Neurology and Centro Clinico Nemo, Fondazione Policlinico Universitario Agostino Gemelli - IRCCS, Rome, Italy.

Received: 8 November 2020 Accepted: 6 June 2021

Published online: 12 June 2021

\section{References}

1. Jerath NU, Shy ME. Hereditary motor and sensory neuropathies: understanding molecular pathogenesis could lead to future treatment strategies. Biochim Biophys Acta. 2015;1852(4):667-78.

2. Barisic N, Claeys KG, Sirotkovic-Skerlev M, Lofgren A, Nelis E, De Jonghe P, Timmerman V. Charcot-Marie-Tooth disease: a clinico-genetic confrontation. Ann Hum Genet. 2008;72(Pt 3):416-41.

3. Bird TD. Charcot-Marie-Tooth (CMT) hereditary neuropathy overview. In: Adam MP, Ardinger HH, Pagon RA, Wallace SE, Bean LJH, Stephens K, Amemiya A, editors. GeneReviews. Seattle: University of Washington; 1993.

4. Previtali SC, Quattrini A, Bolino A. Charcot-Marie-Tooth type 4B demyelinating neuropathy: deciphering the role of MTMR phosphatases. Expert Rev Mol Med. 2007;9(25):1-16.

5. Pareyson D, Stojkovic T, Reilly MM, Leonard-Louis S, Laura M, Blake J, Parman Y, Battaloglu E, Tazir M, Bellatache M, et al. A multicenter retrospective study of charcot-marie-tooth disease type 4B (CMT4B) associated with mutations in myotubularin-related proteins (MTMRs). Ann Neurol. 2019;86(1):55-67.

6. Mammel AE, Delgado KC, Chin AL, Condon AF, Hill JQ, Aicher SA, Wang $Y$, Fedorov LM, Robinson FL. Distinct roles for the Charcot-Marie-Tooth disease-causing endosomal regulators Mtmr5 and Mtmr13 in axon radial sorting and Schwann cell myelination. bioRxiv; 2019. p. 843219.

7. Brennan KM, Bai Y, Shy ME. Demyelinating CMT—what's known, what's new and what's in store? Neurosci Lett. 2015:596:14-26.

8. Lee SM, Chin LS, Li L. Dysregulation of ErbB receptor trafficking and signaling in demyelinating Charcot-Marie-Tooth disease. Mol Neurobiol. 2017;54(1):87-100.

9. Berger P, Young P, Suter U. Molecular cell biology of Charcot-Marie-Tooth disease. Neurogenetics. 2002;4:1-15.

10. Pareyson D, Piscosquito G, Moroni I, Salsano E, Zeviani M. Peripheral neuropathy in mitochondrial disorders. Lancet Neurol. 2013;12(10):1011-24.

11. Carelli V, Chan DC. Mitochondrial DNA: impacting central and peripheral nervous systems. Neuron. 2014;84(6):1126-42.

12. Mancuso M, Orsucci D, Angelini C, Bertini E, Bruno C, Carelli V, Comi GP, Filosto M, Lamperti C, Moggio M, et al. Corrigendum to "Response to: Mitochondrial neuropathy affects peripheral and cranial nerves and is primary or secondary or both" [Neuromuscular Disorders 26/8 (2016) 549]. Neuromuscul Disord. 2017;27(4):e1.

13. Pareyson D, Saveri P, Sagnelli A, Piscosquito G. Mitochondrial dynamics and inherited peripheral nerve diseases. Neurosci Lett. 2015;596:66-77. 
14. Niyazov DM, Kahler SG, Frye RE. Primary mitochondrial disease and secondary mitochondrial dysfunction: importance of distinction for diagnosis and treatment. Mol Syndromol. 2016;7(3):122-37.

15. Alazami AM, Alzahrani F, Bohlega S, Alkuraya FS. SET binding factor 1 (SBF1) mutation causes Charcot-Marie-tooth disease type 4B3. Neurology. 2014;82(18):1665-6.

16. Flusser H, Halperin D, Kadir R, Shorer Z, Shelef I, Birk OS. Novel SBF1 splicesite null mutation broadens the clinical spectrum of Charcot-Marie-Tooth type 4B3 disease. Clin Genet. 2018;94(5):473-9.

17. Manole A, Horga A, Gamez J, Raguer N, Salvado M, San Millan B, Navarro C, Pittmann A, Reilly MM, Houlden H. SBF1 mutations associated with autosomal recessive axonal neuropathy with cranial nerve involvement. Neurogenetics. 2017;18(1):63-7.

18. Nakhro K, Park JM, Hong YB, Park JH, Nam SH, Yoon BR, Yoo JH, Koo H, Jung SC, Kim HL, et al. SET binding factor 1 (SBF1) mutation causes Charcot-Marie-Tooth disease type 4B3. Neurology. 2013;81(2):165-73.

19. Romani M, Mehawej C, Mazza T, Megarbane A, Valente EM. "Fork and bracket" syndrome expands the spectrum of SBF1-related sensory motor polyneuropathies. Neurol Genet. 2016;2(2):e61.

20. Indolfi $G$, lascone M, Remaschi G, Donati MA, Nesti C, Rubegni A, Pezzoli L, Buccoliero AM, Santorelli FM, Resti M. A child with ichthyosis and liver failure. J Pediatr Gastroenterol Nutr. 2017;65(3):e70-3.

21. Bianco A, Martinez-Romero I, Bisceglia L, D’Agruma L, Favia P, Ruiz-Pesini E, Guerriero S, Montoya J, Petruzzella V. Mitochondrial DNA copy number differentiates the Leber's hereditary optic neuropathy affected individuals from the unaffected mutation carriers. Brain. 2016;139(Pt 1):e1.

22. De Michele G, Sorrentino P, Nesti C, Rubegni A, Ruggiero L, Peluso S, Antenora A, Quarantelli M, Filla A, De Michele G, et al. Reversible valproate-induced subacute encephalopathy associated with a MT-ATP8 variant in the mitochondrial genome. Front Neurol. 2018;9:728.

23. Lieto M, Riso V, Galatolo D, De Michele G, Rossi S, Barghigiani M, Cocozza S, Pontillo G, Trovato R, Sacca F, et al. The complex phenotype of spinocerebellar ataxia type 48 in eight unrelated Italian families. Eur J Neurol. 2020;27(3):498-505.

24. Richards S, Aziz N, Bale S, Bick D, Das S, Gastier-Foster J, Grody WW, Hegde $M$, Lyon E, Spector E, et al. Standards and guidelines for the interpretation of sequence variants: a joint consensus recommendation of the American College of Medical Genetics and Genomics and the Association for Molecular Pathology. Genet Med. 2015;17(5):405-24.

25. Senderek J, Bergmann C, Weber S, Ketelsen UP, Schorle H, Rudnik-Schoneborn S, Buttner R, Buchheim E, Zerres K. Mutation of the SBF2 gene, encoding a novel member of the myotubularin family, in Charcot-MarieTooth neuropathy type 4B2/11 p15. Hum Mol Genet. 2003;12(3):349-56.

26. Megarbane A, Dorison N, Rodriguez D, Tamraz J. Multiple cranial nerve neuropathies, microcephaly, neurological degeneration, and "fork and bracket sign" in the MRI: a distinct syndrome. Am J Med Genet A. 2010;152A(9):2297-300

27. Kumar P, Henikoff S, Ng PC. Predicting the effects of coding non-synonymous variants on protein function using the SIFT algorithm. Nat Protoc. 2009;4(7):1073-81.

28. Adzhubei IA, Schmidt S, Peshkin L, Ramensky VE, Gerasimova A, Bork P, Kondrashov AS, Sunyaev SR. A method and server for predicting damaging missense mutations. Nat Methods. 2010;7(4):248-9.

29. Sherry ST, Ward MH, Kholodov M, Baker J, Phan L, Smigielski EM, Sirotkin K. dbSNP: the NCBI database of genetic variation. Nucleic Acids Res. 2001;29(1):308-11.

30. Exome Variant Server, NHLBI GO Exome Sequencing Project (ESP), Seattle, WA. http://evs.gs.washington.edu/EVS/.

\section{Publisher's Note}

Springer Nature remains neutral with regard to jurisdictional claims in published maps and institutional affiliations.
Ready to submit your research? Choose BMC and benefit from:

- fast, convenient online submission

- thorough peer review by experienced researchers in your field

- rapid publication on acceptance

- support for research data, including large and complex data types

- gold Open Access which fosters wider collaboration and increased citations

- maximum visibility for your research: over 100M website views per year

At BMC, research is always in progress.

Learn more biomedcentral.com/submissions 